\title{
Eya1-dependent homeostasis of morphogenetic territories during collective cell migration
}

\author{
Jerónimo R. Miranda-Rodríguez ${ }^{\S}$, Augusto Borges ${ }^{\S}$, Filipe Pinto-Teixeira\#, Indra \\ Wibowo, Hans-Martin Pogoda", Matthias Hammerschmidt", Koichi Kawakami ${ }^{\ddagger}$ and \\ Hernán López-Schier ${ }^{\$ \#^{\star}}$
}

$\S$ Unit of Sensory Biology \& Organogenesis, Helmholtz Zentrum Mu $\square$ nchen, Munich, Germany

II Institute for Developmental Biology, University of Cologne, Cologne, Germany

I School of Technology and Life Sciences, Bandung, Indonesia

\# Center for Developmental Biology, Université Paul Sabatier, Toulouse, France

‡ Laboratory of Molecular and Developmental Biology, National Institute of Genetics

SOKENDAI, 1111 Yata Mishima, Shizuoka 411-8540, Japan

*Corresponding author: hernan.lopez-schier@helmholtz-muenchen.de

\section{SUMMARY}

Tissue remodeling presents an enormous challenge to the stability of intercellular signaling domains. Here we investigate this issue during the development of the posterior lateral line in zebrafish. We find that the transcriptional co-activator and phosphatase Eya1, mutated in the branchio-oto-renal syndrome in humans, is essential for the homeostasis of the Wnt/ $\mathrm{\beta}$ catenin and FGF morphogenetic domains during the collective migration of lateral-line primordial cells. Loss of Eya1 strongly diminishes the expression of Dkk1, expanding Wnt/ $\beta$ catenin activity in the primordium, which in turn abrogates FGFR1 expression. Deficits in Eya1 also abolishes the expression of the chemokine receptor CXCR7b, disrupting primordium migration. These results reinforce the concept that morphogenetic domains in dynamically remodeling tissues are formed by cellular states maintained by continuous signaling. 


\section{INTRODUCTION}

Morphogenesis and pattern formation in multicellular organisms are fundamental for the division of work by different groups of cells. Secreted proteins with morphogenetic capacity can spread across tissues several cell diameters away from their source (Briscoe and Small, 2015; Camacho-Aguilar and Warmflash, 2020). Cellular proliferation, intercalation and migration pose enormous challenges to the spatial control of morphogenetic activity. The Wg/lnt-1 (Wnt) and Fibroblast Growth Factor (FGF) families of secreted signaling proteins are essential for many fundamental biological processes that include embryonic development, organ regeneration and stem-cell physiology (Aulehla et al., 2008; Dodé et al., 2003; Goessling et al., 2009). One example of a remodeling tissue whose development depends on the concerted activity of Wnt/ $\beta$-catenin and FGF signaling is the posterior lateral line of zebrafish (Aman and Piotrowski, 2008; Lecaudey et al., 2008; Nechiporuk and Raible, 2008). This branch of the lateral line develops from a group of just over 100 cells that migrate collectively as a primordium invading the animal's trunk and tail (Ghysen and DamblyChaudière, 2007; Lecaudey and Gilmour, 2006; López-Schier et al., 2004; Metcalfe et al., 1985). The posterior primordium is heterogeneous along its long axis, which runs parallel to the direction of migration. A mitotically active leading area of around 30 cells displays a mesenchymal behavior, whereas trailing cells form epithelial rosettes that are deposited periodically as discrete groups called the neuromasts. The direction and persistence of primordium migration is mediated by the complementary expression pattern of the CXCR4b and CXCR7b chemokine receptors. Leading primordial cells express CXCR4b, whereas CXCR7b is expressed by trailing cells. The expression of both chemokine receptors overlaps in a central transition zone. Rosette deposition involves intercellular signals that act on nonoverlapping parts of the moving cellular collective. The leading region of the primordium activates $\beta$-catenin. The identity of the activating signal is unknown, but it is likely to be a Wnt protein because its activity is controlled by the secreted Wnt inhibitor Dkk1 (Aman and Piotrowski, 2008). Rosettogenesis are under the control of FGF signaling within the trailing region. Elegant genetic experiments and live imaging have revealed a cross-regulation of Wnt/ $\beta$-catenin and FGF morphogens during primordium migration (Fig. 1a) (Aman and Piotrowski, 2008; López-Schier, 2010; Ma and Raible, 2009). However, the complexity of these interactions has made it difficult to clarify how primordial morphogenetic dimensions are monitored and maintained. Here we combine forward- and reverse-genetic analyses with pharmacology, gene expression profiling and quantitative live imaging to study the spatial homeostasis of FGF and Wnt/ $\beta$-catenin signaling. 


\section{RESULTS}

Eya1 is necessary for the development, but not the survival, of the posterior lateral line Because alterations of Wnt/ $\beta$-catenin activity during the migration of the posterior lateralline primordium lead to defects in neuromast deposition (Aman and Piotrowski, 2008), we hypothesized that mutations affecting the number of neuromasts will identify additional factors controlling $\mathrm{Wnt} / \beta$-catenin signaling. Following this rationale, we analyzed zebrafish carrying loss-of-function mutations in Eya1, called dog eared, which have a defective lateral line (Kozlowski et al., 2005). The zebrafish eya1 encodes a protein tyrosine phosphatase with transcriptional activity when in association with the DNA-binding protein So/Six1 (Li et al., 2003; Rayapureddi et al., 2003; Tootle et al., 2003). In addition to a malformed lateral line, recessive mutations in Eya1 produce defects in the adenohypophysis and in the inner ear of the zebrafish (Kozlowski et al., 2005; Nica et al., 2006). We confirmed these previous findings showing that eya1/dog mutant zebrafish develop an average of 4 neuromasts instead of the 8 averaged by wild type animals at 3 days-post-fertilization (dpf) (Fig. 1b-e). We observed identical phenotypes in two independently generated eya1/dog mutant alleles. Because neuromast survival was not affected by the loss of Eya1, the lateral-line defects in the mutants must arise during its initial development.

\section{Eya1 is necessary for the migratory persistence of the primordium}

Zebrafish eya1 is strongly expressed by the lateral-line primordium during migration (Fig. 1f) (Kozlowski et al., 2005; Sahly et al., 1999). To analyze primordium behavior at high spatial and temporal resolution from the onset of migration by confocal videomicroscopy we used the transgenic line $\mathrm{Tg}$ [Cldnb:lyn-EGFP], which expresses membrane-targeted GFP in the lateral-line placodes and primordia (Fig. 1g-h) (Haas and Gilmour, 2006). We found that loss of Eya1 generated primordia that migrated over shorter distances (Fig. 1d-e, g-j). To quantify migration we created kymographs from time-lapse imaging acquired over 440 minutes starting at 32 hours-post-fertilization (hpf), when the primordium is clearly visible in the trunk of the embryo and is fully engaged in the migration process (Fig. 1k-I). Our quantifications showed that the primordium in $\mathrm{Tg}[\mathrm{Cldnb}$ :lyn-EGFP] transgenics moved at a constant velocity of around $80 \mu \mathrm{m} /$ hour at a recording temperature of $27^{\circ} \mathrm{C}$, whereas eya1/dog; $\operatorname{Tg}[\mathrm{Cldnb}$ :IynEGFP] primordia underwent cycles of migration and stalling, averaging $14 \mu \mathrm{m} /$ hour. Mutant eya1/dog lateral lines bore neuromasts that are more anteriorly placed and closely spaced when compared with wild-type controls (Fig. 1d-e). Thus the loss of Eya1 does not block primodium motility, but creates pronounced defects in its otherwise persistent forward migration (Fig. 1k-I). Because defects in cellular viability or mitotic activity within the primordium can decrease neuromast deposition (Aman et al., 2011; Gamba et al., 2010; McGraw et al., 2011; Valdivia et al., 2011), we assessed cellular proliferation in the primordium by performing BrdU incorporation experiments in wild type Tg[Cldnb:lyn-EGFP] 
and eya1/dog;Tg[Cldnb:Iyn-EGFP]. This experiment showed that primordial cells actively divided in both samples (Fig. 2a-b). Because loss of Eya1 decreases cellular viability in several organ primordia, including the zebrafish lateral line (Kozlowski et al., 2005), we assessed apoptosis in wild type and eya1/dog mutant posterior lateral-line primordia by the TUNEL assay (Fig. 2c-d). This experiment showed that whereas most primordial cells in wild type animals remained viable during migration (Fig. 2c), eya1/dog mutants experienced increased apoptosis throughout the entire primordium (Fig. 2d). To ask whether cell death affected neuromast deposition in the mutants, we abrogated apoptosis by injecting a morpholino to p53 (Fig. 2 e,f). The resulting lower levels of apoptosis eya1/dog p53 morphants did not rescue the lateral-line defects associated with the loss of Eya1 (Fig. $2 \mathrm{~g}-\mathrm{h}$ ). Together, these results show that Eya1 is essential for the initial development of the lateral line.

\section{Eya1 is necessary for CXCR7b expression}

To understand the molecular bases of the Eya1 mutant phenotype, we decided to analyze the signaling pathway that governs the collective forward migration of primordial cells: the CXCR4b and CXCR7b chemokine receptors and the Sdf1a/CXCL12 chemokine. We hypothesized that loss of Eya1 does not affect the chemokine because migration occurred along the normal path in the mutants. Indeed, the expression of Sdf1a/CXCL12 was normal along the horizontal myoseptum in the Eya1 mutants (Fig. 3a,b). However, we observed that while CXCR4b remained expressed in the leading zone in Eya1 mutant primordia (Fig. 3c), the expression of CXCR7b was strongly reduced (Fig. 3d). Next, we assessed primordium morphogenesis by labeling Tg[Cldnb:lyn-EGFP] and eya1/dog;Tg[Cldnb:lyn-EGFP] embryos with an antibody to $\mathrm{N}$-cadherin, which highlights the constricted apices of the epithelial cells that form the rosettes. This experiment showed that although epithelialization does occur in the mutants, rosette formation appears defective or delayed (Fig. 3e,f). Detailed observation by confocal videomicroscopy and kymography of eya1/dog;Tg[Cldnb:Iyn-EGFP] specimens showed prominent splitting of the primordium during migration $(30 \%, \mathrm{~N}=10)$ (Fig. $3 \mathrm{~g}, \mathrm{~h}$ ). Interestingly, this phenotype has so far only been associated to the loss of CXCR7b (Valentin et al., 2007). We conclude that the function of Eya1 is intrinsic to the primordium and that its loss causes intermittent primordium migration probably due to strongly reduced $\mathrm{CXCR}$. b expression.

\section{Eya1 is essential for Dkk1 expression and FGF signaling}

Our previous results suggest that Eya1 controls the expression of only a subset of the genes relevant to lateral-line development. We further explored this possibility by profiling gene expression by chromogenic whole-mount in situ hybridization. We focused on the FGF and Wnt/ $\beta$-catenin signaling pathways because their activity domains are always spatially 
segregated in the primordium, and are therefore an ideal dynamic marker for primordial patterning during migration. Fgf3, Lef1 and Sef1 are targets of Wnt/ $\beta$-catenin signaling that are normally expressed by the leading mesenchymal cells (Fig. 4a,c,e) (Ma and Raible, 2009). In the Eya1 mutant embryos, however, the expression domain of Lef1 and Sef1 abnormally extended throughout the primordium and Fgf3 appeared upregulated (Fig. $4 b, d, f)$, suggesting an expansion of the $\beta$-catenin activity domain. We next assessed the expression of Pea3, a bona fide target of FGF signaling, and also of Fgfr1a, whose expression is maintained by a positive feedback loop in the FGF signaling cascade (Aman and Piotrowski, 2008). Both genes are highly expressed by the wild-type primordial trailing cells (Fig. 4g,i). Remarkably, however, their expression was almost completely abolished in eya1/dog mutants (Fig. 4h,j). Collectively, these results indicate that in the absence of Eya1, the primordium experiences a concomitant expansion of $\mathrm{Wnt} / \beta$-catenin activity and a reduction of FGF signaling. This combination of phenotypes has already been reported for zebrafish embryos treated with the FGFR inhibitor SU5402 (Aman and Piotrowski, 2008), suggesting that the loss of Eya1 specifically impairs FGF signaling. The expansion of Wnt/ $\beta$ catenin activity in SU5402-treated embryos results from the decreased expression of the Wnt-signaling inhibitor Dkk1, which is normally found in the primordial transition zone (Fig. $4 \mathrm{k})$. Thus, we assessed the expression of Dkk1 and found that it was almost completely lost in eya1/dog mutants (Fig. 4I).

The expression domains of Lef1 and CXCR7b do not overlap in wild type or eya1/dog mutant primordia (Fig. 5a,b). These observations support previous conclusions that $\mathrm{Wnt} / \beta$-catenin represses $\mathrm{CXCR} 7 \mathrm{~b}$ expression and may explain the restriction of CXCR7b expression to a very few trailing primordial cells by the expanded $W n t / \beta$-catenin signaling domain in eya1/dog mutants (Aman and Piotrowski, 2008). An alternative hypothesis is that Eya1 controls CXCR7b expression independently of Wnt/ $\beta$-catenin. To discriminate between these possibilities, we decided to abrogate $W n t / \beta$-catenin signaling in animals lacking Eya1. If expanded $\mathrm{Wnt} / \beta$-catenin caused CXCR7b repression, the inhibition of $\beta$-catenin activity should rescue CXCR7b expression in the eya1/dog mutants. We first assayed a previously used approach to block Wnt/ $\beta$-catenin signaling based on the mis-expression of Dkk1 by heat shock using the Tg[hsp:Dkk1-EGFP] transgenic line (Aman and Piotrowski, 2008). However, we found that Dkk1 overexpression exhausted the primordium, producing a nonmigrating thin trail of epithelium resembling interneuromast cells (not shown). Identical results were recently reported in the literature (McGraw et al., 2011). Therefore, this approach was not useful for our purpose. To better control the strength and timing of Wnt/ $\mathrm{\beta}$ catenin inhibition, we turned to a pharmacological approach. We used the small molecules IWR-1-endo that stabilizes the $\beta$-catenin destruction complex, and its inactive diastereomer IWR-1-exo as a control (Chen et al., 2009). IWR-1-endo suppressed Lef1 expression in the 
wild type and also in apc and eya1/dog mutant primordia (Fig. 5f-h), indicating that this drug can potently inhibit $\beta$-catenin activity even when the destruction machinery is attenuated by the loss of Apc. The control molecule IWR-1-exo had no discernible effect on gene expression in the primordium (Fig. 5c-e,i-k,o-q). Importantly, neither drug affected primordium size. IWR-1-endo in wild type fish strongly reduced Dkk1 expression (Fig. 5r), but had no effect on CXCR7b (Fig. 5I). Importantly, IWR-1-endo-mediated Wnt inhibition did not rescue the expression of CXCR7b in apc or eya1/dog mutants (Fig. $5 \mathrm{~m}, \mathrm{n}$ ), indicating that CXCR7b repression in eya1/dog does not result from the expanded Wnt/ $\beta$ - catenin activity domain. To test if FGF signaling can rescue CXCR7b expression in eya1/dog mutants, we abrogated Eya1 function and concurrently artificially activated FGF signaling. To this end, we injected a validated translation-blocking morpholino against Eya1 in eggs from the transgenic line $\operatorname{Tg}[$ hsp:CA-FGFR1] that expresses a constitutively active form of the FGFR1 upon heatshock. Ectopic induction of CA-FGFR1 expanded the expression domain of the live sensor of FGF signaling Tg[erm:Gal4;UAS:Kaede] (Esain et al., 2010) (Fig. 6a,b) and also that of Pea3 mRNA (Fig. 6c,d), indicating that FGF signaling was upregulated after heat shock. However, the constitutive FGF signaling in our system did not affect the expression of Dkk1 or CXCR7b in the Eya1 morphants or control embryos (Fig. 6e-n). These results show that Eya1 is necessary for FGF signaling downstream of the FGFR, and that the expansion of the Wnt/ $\beta$ - catenin activity domain in eya1/dog probably results from reduced Dkk1 expression.

\section{DISCUSSION}

Our results show that the activity of the Eya1 is intrinsic to the posterior lateral- line primordium and essential for its forward migration. The loss of Eya1 increases apoptosis throughout the entire primordium. However, we detected specific gene-expression defect in spatially restricted territories of the primordium, which cannot simply be explained by widespread apoptosis. Loss of Eya1 did not affect the directionality of the migration, a phenotype commonly associated to the absence of the chemotactic cue. We confirmed this hypothesis by showing that the expression of Sdf1a/CXCL12 and CXCR4b were normal in Eya1 mutant embryos. Live imaging showed that eya1/dog mutant primordia failed to maintain migratory persistence, and also primordium fragmentations that coincided with migratory failure. This combined phenotype occurs upon loss of CXCR7b (Valentin et al., 2007). Therefore, we reasoned that the migration mutant phenotype might derive from defects in CXCR7b (Fig. 7a). We confirmed this prediction by showing that most of the trailing primordial area failed to express CXCR7b in the absence of Eya1 or Six1b function. Loss of CXCR7b expression could be a consequence of expansions of CXCR4b or of Wnt/ $\beta$ catenin signaling (Aman and Piotrowski, 2008; Dambly-Chaudière et al., 2007). Because CXCR4b expression was not expanded in eya1 mutants, we tested the second possibility by manipulating $W n t / \beta$-catenin activity in wild-type embryos and in those without Eya1 function 
using a pharmacological approach. Our results show that the defective CXCR7b expression in eya1/dog mutant zebrafish is not due to expanded Wnt/ $\beta$-catenin and, consequently, suggest that $\mathrm{Wnt} / \beta$-catenin does not repress CXCR7b expression as previously suggested (Fig. 7b). In addition to the coincidental decrease of CXCR7b expression and the expansion of $\beta$-catenin activity, loss of Eya1 reduced FGF signaling and rosettogenesis in the primordium. This phenotypic combination has been observed upon pharmacological or genetic blockage of FGF signaling (Aman and Piotrowski, 2008; Lecaudey et al., 2008; Nechiporuk and Raible, 2008). Interestingly, while this manuscript was under review, a publication reported that lateral-line rosettogenesis requires the activation of gene expression downstream of FGFR1 (Ernst et al., 2012). Altogether, these results enable us to conclude that the transcriptional activity of Eya1 mediates FGF signaling during lateral-line development (Fig. 7b).

The lateral-line primordium replaces the epithelial trailing cells that it loses with every neuromast deposition by an iterative conversion of mesenchymal progenitors to epithelial rosettes. The mesenchymal-to-epithelial transition zone, formed by around 20 cells, represents the boundary between the Wnt/ $\beta$-catenin and FGF signaling domains. In keeping with the convention, we define these morphogenetic domains "territories" as opposed to "compartments", because the later represents cellular fields that are defined by lineage, whereas the former are dynamically maintained by continuous signaling (Theisen et al., 1996). The rapid movement of mesenchymal cells across the transition zone does not shift significantly the spatial activity domains of Wnt/ $\beta$ - catenin and FGF signaling. How does the lateral-line primordium maintains signaling territories and persistent migration while undergoing periodic loss of trailing $\mathrm{FGF}(+) / \mathrm{CXCR} 7 \mathrm{~b}(+)$ cells, and expansion and movement of leading Wnt/B-catenin(+)/CXCR4b(+) cells? The expression of Dkk1 in the transition zone establishes the rear boundary of $\beta$-catenin activity. Also, $\mathrm{Wnt} / \beta$ - catenin signaling promotes FGF activity within the epithelial area, which is achieved through a $\beta$-catenin-dependent expression of the FGFR ligands Fgf3 and Fgf10. In turn, FGF signaling promotes the expression of FGFR1 by a positive feedback loop, and restricts Wnt/ 3 -catenin signaling by activating Dkk1 expression (Fig. 1a) (Aman and Piotrowski, 2008; Ma and Raible, 2009). Although this model is consistent with the spatial expression profile of most genes in the primordium, it cannot explain that of Dkk1 because the strongest FGF output is located within the entire epithelial zone, which does not express Dkk1. Therefore, although Dkk1 represents a central hub in the regulatory network that governs the homeostasis of FGF and Wnt/ $\beta$-catenin signaling during primordium migration, how its expression is dynamically controlled remains unknown. Because Dkk1 expression appears tightly linked to the mesenchymal-to-epithelial transition zone, a combinatorial activity of Wnt/ $\beta$-catenin and FGF activity could suffice to govern Dkk1 expression in space and time. This idea predicts that a 
combined expansion of FGF and Wnt/ 3 -catenin signaling should also expand the expression domain of Dkk1, which has been observed in apc mutant fish. However, our ectopic activation of FGF signaling was not sufficient to expand Dkk1 expression to the Wnt/ßcatenin territory. This discrepancy may be explained by the observation that loss of Apc and ectopic FGF signaling do not lead to equivalent results (Aman and Piotrowski, 2008; Lecaudey et al., 2008; Nechiporuk and Raible, 2008). Abnormally persistent $\beta$-catenin in apc mutants expands the spatial domain and temporal duration of Wnt/ $\beta$ - catenin signaling and, concurrently, expands the spatial domain of FGF signaling. By contrast, the sole expansion of FGF signaling does not change the profile of Wnt/ $\beta$-catenin signaling. Thus, we would like to offer a new model for the dynamic maintenance of Dkk1 at the transition zone (Fig. 7c). Our model suggests that FGF strength and time-dependent Wnt/ $\beta$-catenin must coincide to initiate and maintain Dkk1 expression. This new model, although speculative at present, best accommodates the collective experimental evidence. In concluding, the results of this study refine our understanding of the complex regulatory interaction between FGF and Wnt/ $\beta$ catenin signaling during the collective migration of posterior lateral-line cells. Further dissection of the genetic network that controls the homeostasis of morphogenetic territories in the lateral-line primordium will yield insights on the general mechanisms that underlie rapid and orderly cell-fate transitions in a wide variety of biological processes.

\section{MATERIALS AND METHODS}

\section{Zebrafish animals and strains}

Fish used were maintained under standardized conditions and experiments were performed in accordance with protocols approved by the PRBB Ethical Committee of Animal Experimentation. Eggs were collected from natural spawning and maintained at $28.5^{\circ} \mathrm{C}$. Embryos were staged by hours post fertilization (hpf). Two eya1/dog mutant alleles were used in this study, aalt22744 and $\operatorname{dog}^{\wedge} \operatorname{tm} 90$ (Kozlowski et al., 2005; Nica et al., 2006). Embryos were genotyped according to Kozlowski et al. (Kozlowski et al., 2005). Both alleles showed identical phenotypes in all results obtained. In this study we mainly used the $d^{\prime} g^{\wedge} t m 90$ allele. The apc allele was mor (Haramis et al., 2006). Transgenic lines used were Tg[SqET4]. Tg[SqET10], Tg[SqET20] (Parinov et al., 2004), Tg[Cldnb:IynEGFP] (Haas and Gilmour, 2006), Tg[hs70:CA-FGFR1] (Lee et al., 2005), Tg[hs70:EGFP-Dkk1] (Stoick-Cooper et al., 2007), Tg[erm:Gal4;UAS:Kaede] (Esain et al., 2010). Heat-shock treatments were 
performed by immersing embryos in the $\mathrm{E} 3$ medium in $2-\mathrm{ml}$ tubes in a water bath at $39^{\circ} \mathrm{C}$ for 20 minutes.

\section{Drug treatments}

$10 \mathrm{mM}$ stock solutions of IWR-1 drugs (Millipore) diluted in DMSO were used. Twenty-four zebrafish embryos were dechorionated and allowed to develop in E3 medium supplemented with 75-100 $\mu \mathrm{M}$ IWR-1-endo or IWR-1-exo or in 0.1\% DMSO as a control.

\section{Morpholino antisense oligonucleotides}

cRNAs were synthesized using mMessage mMachine (Ambion) according to manufacturer's instructions. Morpholinos that were used in this study were: MO1Eya1 (5'AAACAAAGATGATAGACCTACTTCC-3', (Kozlowski et al., 2005).

\section{BrdU treatment}

10 mM 5-Bromo-2'-deoxyuridine (BrdU, B5002, Sigma) stock solution in DMSO was diluted to $10 \mu \mathrm{M}$ in E3 medium and used to soak embryos. BrdU was incorporated into newly synthesized DNA in the S-phase; therefore, it functioned as a marker for cell proliferation. Embryos at $24 \mathrm{hpf}$ were dechorionated and allowed to develop in this solution until desired stages. Embryos were fixed in $4 \%$ Paraformaldehyde (PFA) overnight at $4^{\circ} \mathrm{C}$ and then used for immunohistochemistry.

\section{TUNEL assay}

Apoptosis in the migrating primordium was identified using terminal transferase-mediated dUTP nick end-labeling (TUNEL) assay according to manufacturer's instruction with minor modifications (In situ Cell Death Detection Kit, TMR Red, Roche). Embryos at 30-42 hpf were dechorionated and fixed in $4 \%$ PFA overnight at $4{ }^{\circ} \mathrm{C}$, then stored in $100 \%$ methanol at $-20^{\circ} \mathrm{C}$ for at least 1 day. They were rehydrated, permeabilized in $10 \mu \mathrm{g} / \mathrm{ml}$ Proteinase $\mathrm{K}$ in $0.1 \%$ PBSTw and post-fixed in 4\% PFA then washed several times in $0.1 \%$ PBSTw. Embryos were then incubated in fresh TUNEL buffer for $1 \mathrm{~h}$ followed by incubation in the TUNEL reaction mix for $3 \mathrm{hrs}$ at $37^{\circ} \mathrm{C}$ in dark. As negative control, embryos were incubated in TUNEL buffer only. As positive control, embryos were first incubated in polymerase chain reaction buffer containing $3 \mathrm{U} / \mathrm{ml}$ DNase I recombinant (Roche) for $1 \mathrm{~h}$ at $37^{\circ} \mathrm{C}$ before incubation in TUNEL reaction mix. After the reaction, embryos were washed several times in $0.1 \%$ PBStw at room temperature and stored in $0.1 \%$ PBSTw containing Vectashiled mounting medium with DAPI (VectorLabs).

\section{Whole-mount immunohistochemistry and in situ hybridization}

For immunohistochemistry and ISH, staged embryos were dechorionated and fixed in $4 \%$ PFA for overnight at $4^{\circ} \mathrm{C}$ and washed several times with $0.1 \%$ Tween-20-containing Phosphate Buffer Saline $(0.1 \%$ PBSTw). For immunohistochemistry, larvae were immediately blocked in 10\% Bovine Serum Albumin (BSA) for at least 2 hours. Incubation with primary antibody was done overnight at $4^{\circ} \mathrm{C}$. Primary antibodies and monoclonal 
antibodies were used at the following dilutions: mouse monoclonal antibody anti-BrdU, 1:100 (Upstate), rabbit anti-N-cadherin, 1/500. Texas red-labeled donkey anti-mouse and -rabbit and Cy5-labeled donkey anti-mouse and - rabbit immunoglobulin secondary antibodies (Jackson ImmunoResearch) were used at 1/150. For BrdU labeling detection, before blocking and applying primary antibody anti-mouse, additional steps were needed to permeabilize the nuclear membrane and to denature DNA strands. Larvae were incubated with $10 \mu \mathrm{g} / \mathrm{ml}$ Proteinase $\mathrm{K}$ in $0.1 \%$ PBSTw for $20 \mathrm{~min}$ at room temperature. Immediately afterwards, larvae were post-fixed in 4\% PFA for $15 \mathrm{~min}$ and washed several times in $0.1 \%$ PBSTw. To denature DNA, samples were incubated in fresh $2 \mathrm{~N} \mathrm{HCl}$ for $1 \mathrm{~h}$ and washed several times in $0.1 \%$ PBSTw. Samples were blocked in BrdU blocking solution for at least 2 hrs at RT and then incubated in BrdU blocking solution containing the antibodies at $4^{\circ} \mathrm{C}$. For $\mathrm{ISH}$, antisense digoxigenin- and fluorescein-labeled riboprobes were synthesized according to manufacturer's instructions (Roche) by using T7/SP6/T3 RNA polymerases. Probes used were: Sdf1a, Cxcr7b, Cxcr4b, Fgfr1a, Fgf3, Pea3, Sef1, Lef1, Dkk1, and Eya1. Whole-mount two-color fluorescence ISH was performed using anti-DIG and -fluorescein POD antibodies (Roche) and Tyramide Signal Amplification (TSA, PerkinElmer) to detect the riboprobes. Larvae were mounted in $0.1 \%$ PBSTw with Vectashield/DAPI (1/100, Vector Labs.)

\section{Imaging and time-lapse videomicroscopy}

For whole-mount ISH, embryos were deyolked, flat mounted and photographed with a Olympus BX61 microscope using 20X or 40X dry objectives with transmission light. Whole embryo images were acquired on a Leica MZ10 stereomicroscope. Fluorescent images were acquired using either a Leica SP5 or SPE microscope using 20X dry objective or 40X oilimmersion objective as previously reported (Faucherre et al., 2009). Images were processed using Imaris and/or ImageJ software packages, and assembled with Adobe Photoshop CS2, Adobe Illustrator CS2, and Macromedia FreeHand MX. For time-lapse imaging, staged and dechorionated embryos were anesthetized with tricaine and mounted in 0.8-1\% low-meltingpoint agarose on a glass-bottom culture dish (MatTek) as previously described (Wibowo et al., 2011). Z-stack series were acquired every 4-10 min using a 20X dry objective of Leica SPE or SP5 confocal microscope. All movies were processed with the Imaris or ImageJ software packages. An unpaired two-tailed $T$ test with Welch's correction was used to compare the position of neuromast $L 4$ in eya1/dog mutants and wild-type siblings. A two-way ANOVA with replication was used to compare neuromast distribution in wt and Six1b mutants. Statistics were performed using the GraphPad Prism software and Excel running QI Macros.

\section{ACKNOWLEDGEMENTS}


We are indebted to A-P. Haramis, K. Poss, G. Weidinger, J. Ghislain, and T. Whitfield for their gift of zebrafish lines and DNAs. This work was funded by the Helmholtz Association to HL-S.

\section{REFERENCES}

Aman, A., Nguyen, M., and Piotrowski, T. (2011). Wnt/beta-catenin dependent cell proliferation underlies segmented lateral line morphogenesis. Dev Biol 349, 470-482. Aman, A., and Piotrowski, T. (2008). Wnt/beta-catenin and Fgf signaling control collective cell migration by restricting chemokine receptor expression. Dev Cell 15, 749-761.

Aulehla, A., Wiegraebe, W., Baubet, V., Wahl, M.B., Deng, C., Taketo, M., Lewandoski, M., and Pourquié, O. (2008). A beta-catenin gradient links the clock and wavefront systems in mouse embryo segmentation. Nat Cell Biol 10, 186-193.

Bessarab, D.A., Chong, S.W., and Korzh, V. (2004). Expression of zebrafish six1 during sensory organ development and myogenesis. Dev Dyn 230, 781-786.

Bricaud, O., and Collazo, A. (2006). The transcription factor six1 inhibits neuronal and promotes hair cell fate in the developing zebrafish (Danio rerio) inner ear. J. of Neurosci. 26, 10438-10451.

Briscoe, J. and Small, S. (2015) Morphogen rules: design principles of gradient-mediated embryo patterning. Development 142(23):3996-4009

Camacho-Aguilar, E. and Warmflash A., (2020) Insights into mammalian morphogen dynamics from embryonic stem cell systems. Curr Top Dev Biol. 137:279-305.

Chen, B., Dodge, M.E., Tang, W., Lu, J., Ma, Z., Fan, C.W., Wei, S., Hao, W., Kilgore, J., Williams, N.S., et al. (2009). Small molecule-mediated disruption of Wnt-dependent signaling in tissue regeneration and cancer. Nat Chem Biol 5, 100-107.

Dahmann, C., Oates, A.C., and Brand, M. (2011). Boundary formation and maintenance in tissue development. Nat Rev Genet 12, 43-55.

Dambly-Chaudière, C., Cubedo, N., and Ghysen, A. (2007). Control of cell migration in the development of the posterior lateral line: antagonistic interactions between the chemokine receptors CXCR4 and CXCR7/RDC1. BMC Dev Biol 7, 23. 
Dessaud, E., Yang, L.L., Hill, K., Cox, B., Ulloa, F., Ribeiro, A., Mynett, A., Novitch, B.G., and Briscoe, J. (2007). Interpretation of the sonic hedgehog morphogen gradient by a temporal adaptation mechanism. Nature 450, 717-720.

Dodé, C., Levilliers, J., Dupont, J.M., De Paepe, A., Le Du, N., Soussi-Yanicostas, N., Coimbra, R.S., Delmaghani, S., Compain-Nouaille, S., Baverel, F., et al. (2003). Loss-offunction mutations in FGFR1 cause autosomal dominant Kallmann syndrome. Nat Genet 33, 463-465.

Ernst, S., Liu, K., Agarwala, S., Moratscheck, N., Avci, M.E., Dalle Nogare, D., Chitnis, A.B., Ronneberger, O., and Lecaudey, V. (2012). Shroom3 is required downstream of FGF signalling to mediate proneuromast assembly in zebrafish. Development 139, 4571-4581.

Esain, V., Postlethwait, J.H., Charnay, P., and Ghislain, J. (2010). FGF-receptor signalling controls neural cell diversity in the zebrafish hindbrain by regulating olig2 and sox9. Development 137, 33-42.

Faucherre, A., Pujol-Martí, J., Kawakami, K., and López-Schier, H. (2009). Afferent neurons of the zebrafish lateral line are strict selectors of hair-cell orientation. PloS one 4 , e4477.

Gamba, L., Cubedo, N., Lutfalla, G., Ghysen, A., and Dambly-Chaudière, C. (2010). Lef1 controls patterning and proliferation in the posterior lateral line system of zebrafish. Dev Dyn 239, 3163-3171.

Ghysen, A., and Dambly-Chaudière, C. (2007). The lateral line microcosmos. Genes Dev 21, 2118-2130.

Goessling, W., North, T.E., Loewer, S., Lord, A.M., Lee, S., Stoick-Cooper, C.L., Weidinger, G., Puder, M., Daley, G.Q., Moon, R.T., et al. (2009). Genetic interaction of PGE2 and Wnt signaling regulates developmental specification of stem cells and regeneration. Cell 136, 1136- 1147.

Grigoryan, T., Wend, P., Klaus, A., and Birchmeier, W. (2008). Deciphering the function of canonical Wnt signals in development and disease: conditional loss- and gain-of-function mutations of beta-catenin in mice. Genes Dev 22, 2308-2341.

Haas, P., and Gilmour, D. (2006). Chemokine signaling mediates self-organizing tissue migration in the zebrafish lateral line. Dev Cell 10,673-680. 
Haramis, A.P., Hurlstone, A., van der Velden, Y., Begthel, H., van den Born, M., Offerhaus, G.J., and Clevers, H.C. (2006). Adenomatous polyposis coli-deficient zebrafish are susceptible to digestive tract neoplasia. EMBO Rep 7, 444-449.

Kozlowski, D.J., Whitfield, T.T., Hukriede, N.A., Lam, W.K., and Weinberg, E.S. (2005). The zebrafish dog-eared mutation disrupts Eya1, a gene required for cell survival and differentiation in the inner ear and lateral line. Dev Biol 277, 27-41.

Lecaudey, V., Cakan-Akdogan, G., Norton, W.H., and Gilmour, D. (2008). Dynamic Fgf signaling couples morphogenesis and migration in the zebrafish lateral line primordium. Development 135, 2695-2705.

Lecaudey, V., and Gilmour, D. (2006). Organizing moving groups during morphogenesis. Curr Opin Cell Biol 18, 102-107.

Lee, Y., Grill, S., Sanchez, A., Murphy-Ryan, M., and Poss, K.D. (2005). Fgf signaling instructs position-dependent growth rate during zebrafish fin regeneration. Development 132 , 5173-5183.

Li, X., Oghi, K.A., Zhang, J., Krones, A., Bush, K.T., Glass, C.K., Nigam, S.K., Aggarwal, A.K., Maas, R., Rose, D.W., et al. (2003). Eya protein phosphatase activity regulates Six1Dach-Eya transcriptional effects in mammalian organogenesis. Nature 426, 247-254.

López-Schier, H. (2010). Fly fishing for collective cell migration. Curr Opin Genet Dev 20, 428- 432.

López-Schier, H., Starr, C.J., Kappler, J.A., Kollmar, R., and Hudspeth, A.J. (2004). Directional cell migration establishes the axes of planar polarity in the posterior lateral-line organ of the zebrafish. Developmental cell 7, 401-412.

Ma, E.Y., and Raible, D.W. (2009). Signaling pathways regulating zebrafish lateral line development. Curr Biol 19, R381-386.

McGraw, H.F., Drerup, C.M., Culbertson, M.D., Linbo, T., Raible, D.W., and Nechiporuk, A.V. (2011). Lef1 is required for progenitor cell identity in the zebrafish lateral line primordium. Development 138, 3921-3930.

Metcalfe, W.K., Kimmel, C.B., and Schabtach, E. (1985). Anatomy of the posterior lateral line system in young larvae of the zebrafish. The Journal of comparative neurology 233, 377389. 
Nechiporuk, A., and Raible, D.W. (2008). FGF-dependent mechanosensory organ patterning in zebrafish. Science 320, 1774-1777.

Nguyen, D.X., Chiang, A.C., Zhang, X.H., Kim, J.Y., Kris, M.G., Ladanyi, M., Gerald, W.L., and Massagué, J. (2009). WNT/TCF signaling through LEF1 and HOXB9 mediates lung adenocarcinoma metastasis. Cell 138, 51-62.

Nica, G., Herzog, W., Sonntag, C., Nowak, M., Schwarz, H., Zapata, A.G., and Hammerschmidt, M. (2006). Eya1 is required for lineage-specific differentiation, but not for cell survival in the zebrafish adenohypophysis. Dev Biol 292, 189-204.

Parinov, S., Kondrichin, I., Korzh, V., and Emelyanov, A. (2004). Tol2 transposonmediated enhancer trap to identify developmentally regulated zebrafish genes in vivo. Dev Dyn 231, 449- 459.

Rayapureddi, J.P., Kattamuri, C., Steinmetz, B.D., Frankfort, B.J., Ostrin, E.J., Mardon, G., and Hegde, R.S. (2003). Eyes absent represents a class of protein tyrosine phosphatases. Nature 426, 295-298.

Sahly, I., Andermann, P., and Petit, C. (1999). The zebrafish eya1 gene and its expression pattern during embryogenesis. Development genes and evolution 209, 399-410.

Stoick-Cooper, C.L., Weidinger, G., Riehle, K.J., Hubbert, C., Major, M.B., Fausto, N., and Moon, R.T. (2007). Distinct Wnt signaling pathways have opposing roles in appendage regeneration. Development 134, 479-489.

Theisen, H., Haerry, T.E., O'Connor, M.B., and Marsh, J.L. (1996). Developmental territories created by mutual antagonism between Wingless and Decapentaplegic. Development 122, 3939-3948.

Tootle, T.L., Silver, S.J., Davies, E.L., Newman, V., Latek, R.R., Mills, I.A., Selengut, J.D., Parlikar, B.E., and Rebay, I. (2003). The transcription factor Eyes absent is a protein tyrosine phosphatase. Nature 426, 299-302.

Valdivia, L.E., Young, R.M., Hawkins, T.A., Stickney, H.L., Cavodeassi, F., Schwarz, Q., Pullin, L.M., Villegas, R., Moro, E., Argenton, F., et al. (2011). Lef1-dependent Wnt/betacatenin signalling drives the proliferative engine that maintains tissue homeostasis during lateral line development. Development 138, 3931-3941. 
Valentin, G., Haas, P., and Gilmour, D. (2007). The chemokine SDF1a coordinates tissue migration through the spatially restricted activation of Cxcr7 and Cxcr4b. Curr Biol 17, 10261031.

van Amerongen, R., and Nusse, R. (2009). Towards an integrated view of Wnt signaling in development. Development 136, 3205-3214.

Wibowo, I., Pinto-Teixeira, F., Satou, C., Higashijima, S., and López-Schier, H. (2011). Compartmentalized Notch signaling sustains epithelial mirror symmetry. Development 138 , 1143-1152.

\section{FIGURE LEGENDS}

Figure 1. The lateral-line primordium in the wild type and eya1/dog mutant zebrafish. (a)Schematic representation of the genetic network that maintains primordium compartmentalization and migratory persistence, as it is currently known. The arrows indicate activation and the T-bars inhibition. The question marks indicate that the identity of the activators or inhibitors is unknown. (b,c) Wild type (b) and eya1/dog mutants (c) Tg[CldnB:lynEGFP] larvae at $72 \mathrm{hpf}$. (d,e) Plot of the distance (in $\mu \mathrm{m}$ ) between the caudal limit of the otic vesicle and the average number of deposited neuromasts in eya1/dog mutants and wild-type siblings at $3 \mathrm{dpf}$ (meants.d.). (f) Chromogenic whole-mount in situ hybridization (WM- ISH) showing Eya1 expression in the posterior lateral-line primordium (black arrowhead in d). (g-h) Low magnification confocal images of a Tg[CldB:IynEGFP] embryo (g) and a eya1/dog;Tg[CldB:IynEGFP] (h) at 32 hpf showing the delayed migration of the primordium (white arrowhead) in the mutants. (i,j) High magnification confocal images of a wild type (i) and mutant (j) primordium. At 195 minutes of migration, the wild-type primordium has deposited one pro-neuromast, whereas the eya1/dog primordium failed to do so even after 440 minutes. (k-I) Kymography of wild type (k) and eya1/dog primordia in the Tg[CldB:lynEGFP] transgenic background (I). The wild-type primordium shows regular speed and periodical deposition of two pro-neuromasts, whereas the eya1/dog mutant primordium shows irregular speed and no neuromast deposition.

Figure 2. Mitotic activity or apoptosis cannot explain the lateral-line defects in eya1/dog. $(\mathbf{a}, \mathbf{b})$ Cell proliferation analysis of wild type and eya1/dog primordial. BrdU incorporation (red) on $\mathrm{Tg}[\mathrm{CldB}: \mathrm{lyn} E \mathrm{GFP}]$ embryos (green) shows no obvious differences in S-phase completion between wild type (a) and eya1/dog mutant embryos (b). (c-f) Cell viability analysis of wild type (c) and eya1/dog primordia (d) and injected with injected with a 
p53 morpholino $(\mathbf{e}, \mathbf{f})$ by the TUNEL assay (red) on Tg[CldB:lynEGFP] embryos (green) counterstained with the nuclear dye DAPI (blue). Increased of apoptosis is observed in the eya1/dog mutant primordium (dotted outline on the lowed panels) (d). The resulting decrease in apoptosis in p53 injected mutants did not rescue the lateral-line defects associated with the loss of Eya1 (e-h). (g) Quantification of the number of neuromasts at $72 \mathrm{hpf}$ in wild type (blue bars) and eya1/dog (green bars). The average number of neuromasts were counted in non-injected and MOp53- injected Tg[CldnB:lynEGFP] controls (blue bars) and eya1/dog;Tg[CldnB:lynEGFP] (green bars). The total number of neuromasts did not differ between non-injected and MOp53-injected wild type embryos. The data is represented as mean \pm standard deviation (s.d.). ( $N=23, P<0,001$, Unpaired two-tailed $T$ test with Welch's correction). (h) The average distance of neuromast L4 to the otic lobe (used as morphological landmark) was used as a proxy to the extent of migration in non-injected and MOp53-injected Tg[CldnB:lynEGFP] controls (blue bars) and eya1/dog;Tg[CldnB:lynEGFP] (green bars). The position of L4 did not differ between non- injected and MOp53-injected wild type or dog/eya1 mutant embryos. The position of the neuromasts in eya1/dog is significantly shifted rostrally, as revealed by the position of neuromast $L 4(\mathrm{~N}=18-23, \mathrm{P}<0,001$, Unpaired two-tailed T test with Welch's correction).

Figure 3. Loss of CXCR7b expression and primordium splitting in eya1/dog mutants. (a,d) Two-color fluorescent whole-mount in situ hybridization of Sdf1a/CXCL12 (red) (c,d) CXCR4b (green) and CXCR7b (red) gene-expression profiles in wild type (a,c) and eya1/dog mutants (b,d), counterstained with DAPI (blue) to reveal the nuclei for better identification of the primordium (white dotted outline). It shows that the Sdf1a/CXCL12 gene (red) is expressed along the horizontal myoseptum in the wild type and eya1/dog mutants. The CXCR4b gene is strongly expressed in the leading region of primordium in both in wild type and eya1/dog mutants. The expression of CXCR7b, however, is strong in the trailing region of the wild-type primordium (overlapping with CXCR4b), but almost completely lost in eya1/dog mutants, as it is restricted to the very end of the trailing region and never overlaps with CXCR4b. (e,f) Antibody labelling of N-cadherin (red) to highligth adherens junction and the apical constriction of rosettes in Tg[CldB:lynEGFP] embryos (green). Apical constriction of the rosettes are evident in the wild type sample (e), but they are disrupted in eya1/dog mutants (f). (g) Still images of 170 minutes time lapse videomicroscopy and (h) kymography of a eya1/dog mutant showing a transient splitting of the leading zone of the primordium. The four timeframes $(\mathbf{g})$ correspond to the dark hole in the kymograph (h) (asterisks on the black overline in $\mathbf{g}$ and white vertical line in $\mathbf{h})$.

Figure 4. Loss of Eya1 function de-regulates gene expression in the primordium. (a-f) WM-ISH for Fgf3, Sef and Lef1 genes, which are normally expressed in cells of leading 
zone (a,c,e) while in eya1/dog mutants Fgf3 (b) expression is up-regulated, and Sef1 (d) and Lef1 (f) are expanded throughout the primordium. Fgfr1a and Pea3 are normally expressed in the trailing epithelial cells $(\mathbf{g}, \mathbf{i})$, whereas their expression is almost completely lost in eya1/dog mutants. (h,j). Dkk1 expression is normally strong in the transition zone (k), while in eya1/dog mutants Dkk1 expression is lost (I). A black dotted line identifies the primordium in each panel. All panels on the left correspond to wild type, and those on the right to eya1/dog mutants.

Figure 5. Loss of Eya1 affects CXCR7b expression independently of Wnt/ $\beta$-catenin. $(\mathbf{a}, \mathbf{b})$ TC-FISH of Lef1 (red) and CXCR7b (green) shows non-overlapping domains of expression in wild type (a) and eya1/dog mutants (b). (c-h) WM-ISH shows that IWR-endo inhibits Wnt/ $\beta$-catenin signaling as assessed by Lef1 expression in wild type (f), apc (g) and eya1/dog (h) mutant zebrafish lateral-line primordia, whereas the inactive IWR-exo causes no effects (c-e). (i-n) Neither IWR-endo, nor IWR-exo revert the loss of CXCR7b in apc (j,m) or eya1/dog $(\mathbf{k}, \mathbf{n})$ mutants. (o-t) IWR-endo reduces the expression of Dkk1 in the wild type condition ( $\mathbf{r}$ ) and diminishes dkk1 expansion in apc (s) mutants, but does not rescue dkk1 expression in eya1/dog (t) mutants. IWR-exo causes no effect on these genes expession profiles (o-q). A black dotted outline identifies the primordium in each panel.

\section{Figure 6. Eya1 is necessary for FGF signaling.}

$(\mathbf{a}, \mathbf{b})$ Fluorescent images of the posterior lateral-line primodium in the double transgenic line Tg[hsp70:ca-Fgfr1;erm:Gal4-UAS:kaede] (green) counterstained with DAPI (blue) without (a) and with (b) heat shock. Note the conspicuous absence of Kaede in the front of the primordium in the control (a) and its expansion in upon heat shock (b). (c,d) WM-ISH for Pea3 in the posterior lateral-line primodium in wild type controls (c) and Tg[hsp70:ca-Fgfr1] transgenics (d). Note the expression of Pea3 in the trailing area of the primordium in the control and its expansion throughout the primordium upon heat shock in the transgenics. These results show that the Tg[hsp70:ca-Fgfr1] transgenic line broadly activates FGF signaling upon heat shock. (e-j) WM-ISH to CXCR7b in the posterior lateral-line primodium in wild type controls (e,g), Eya1 morphants (h), Tg[hsp70:ca-Fgfr1] transgenics (i) and Tg[hsp70:ca-Fgfr1] transgenics + Eya1 morphants (j). They show that overactivation of FGF signaling does not expand the expression domain of CXCR7b (i), and does not rescue the down-regulation of CXCR7b expression upon loss of Eya1 $(\mathbf{g}, \mathbf{h}, \mathbf{j})$. WM-ISH for Dkk1 in the posterior lateral-line primodium in wild type controls (k), Eya1 morphants (I), Tg[hsp70:caFgfr1] transgenics $(\mathbf{m})$ and Tg[hsp70:ca-Fgfr1] transgenics + Eya1 morphants (n). They show that overactivation of FGF signaling neither expands the expression domain of Dkk1 $(\mathbf{m})$, nor it rescues its expression upon loss of Eya1 $(\mathbf{k}, \mathbf{l}, \mathbf{n})$. A black dotted outline identifies the primordium. 
Figure 7. Summary of the results and new model of the posterior lateral-line primordium. (a) Diagram of the primordium (ovals). Signaling territories and geneexpression patterns are color coded in wild type (a) and Eya1 loss-of-function (b). (b) A new model of the genetic network organizing the posterior lateral-line primordium places Eya1 downstream of FGFR. The scheme indicates that two feed-forward loops $(\mathrm{Wnt} / \beta$-catenin to FGF via the activation of FGF3/10; and FGF to Wnt/ß-catenin, via the activation of dkk1) and one positive feedback loop (FGF signaling autoactivation) dynamically maintain the spatial domains of activity of Wnt/ $\beta$ - catenin and FGF signaling during primordium migration. The three green question marks indicate that the activating or inhibiting signals are currently unknown. (c) An hypothetical model proposing that only the cells that coincidentally reach certain threshold of FGF and Wnt/ $\beta$-catenin signaling will be able to initiate and maintain Dkk1 expression. 
bioRxiv preprint doi: https://doi.org/10.1101/2021.01.27.428404; this version posted January 27, 2021. The copyright holder for this preprint (which was not certified by peer review) is the author/funder, who has granted bioRxiv a license to display the preprint in perpetuity. It is made available under aCC-BY-NC-ND 4.0 International license.
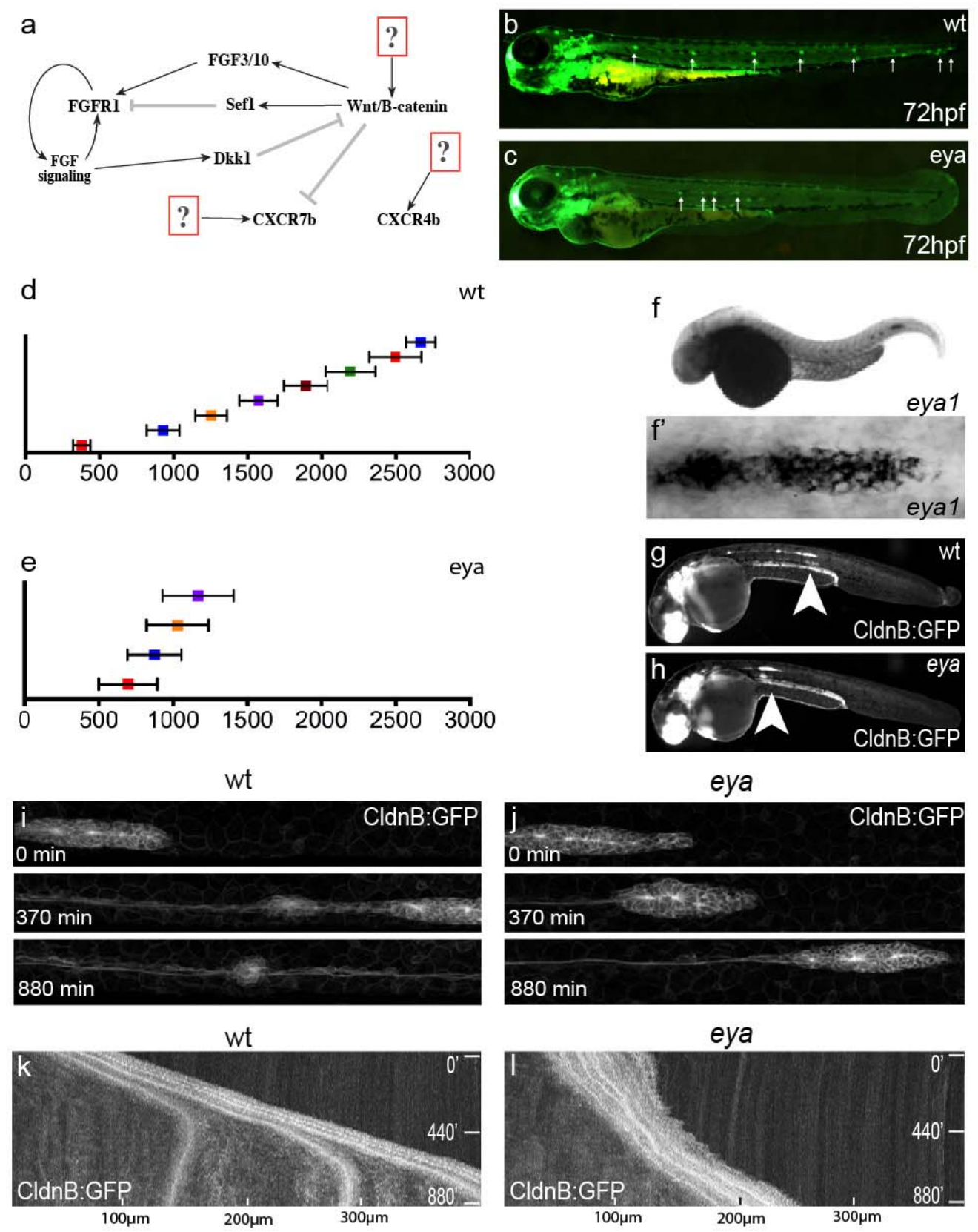

Figure 1 
bioRxiv preprint doi: https://doi.org/10.1101/2021.01.27.428404; this version posted January 27, 2021. The copyright holder for this preprint

(which was not certified by peer review) is the author/funder, who has granted bioRxiv a license to display the preprint in perpetuity. It is made available under aCC-BY-NC-ND 4.0 International license.
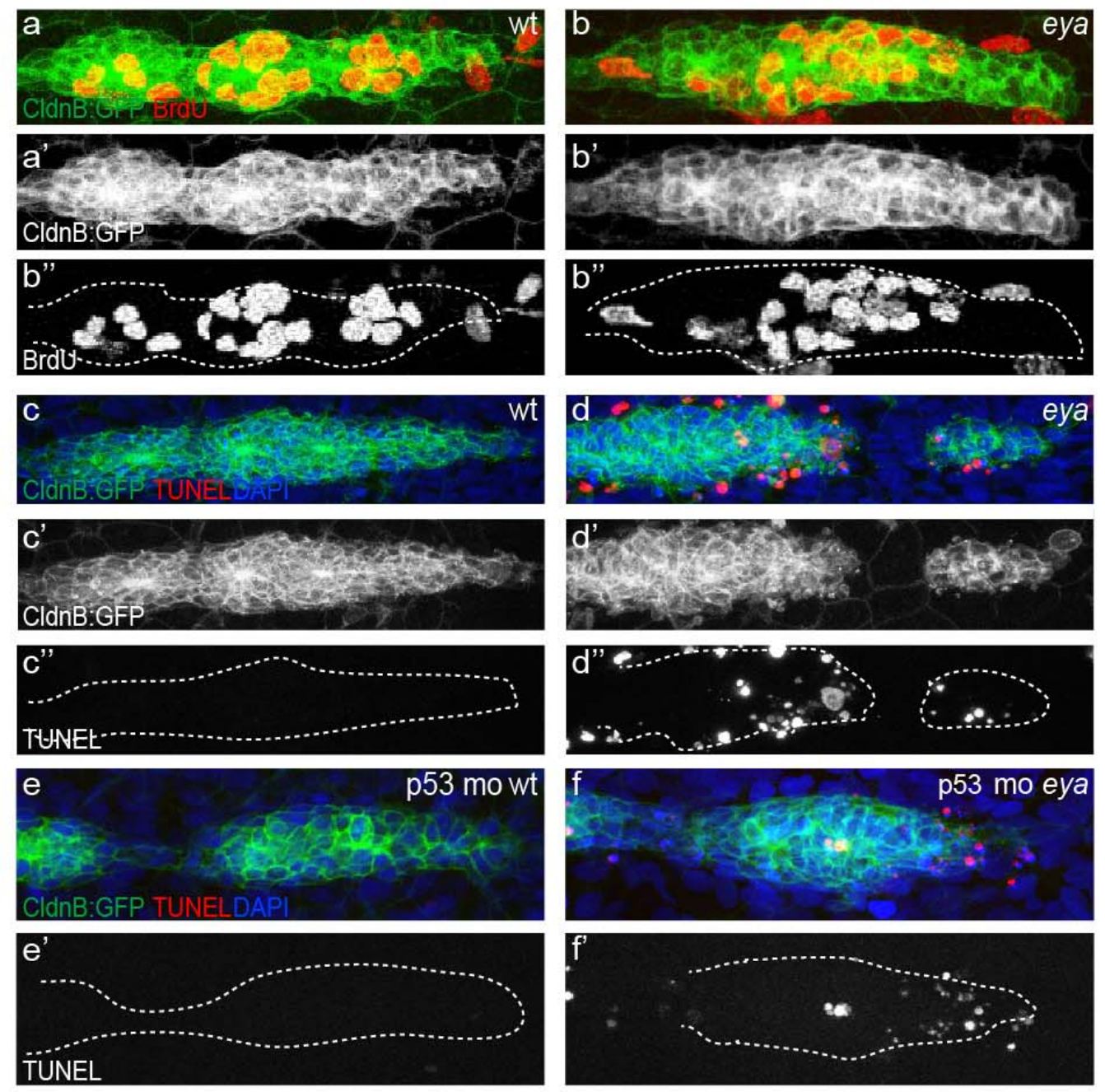

$\mathrm{g}$

h
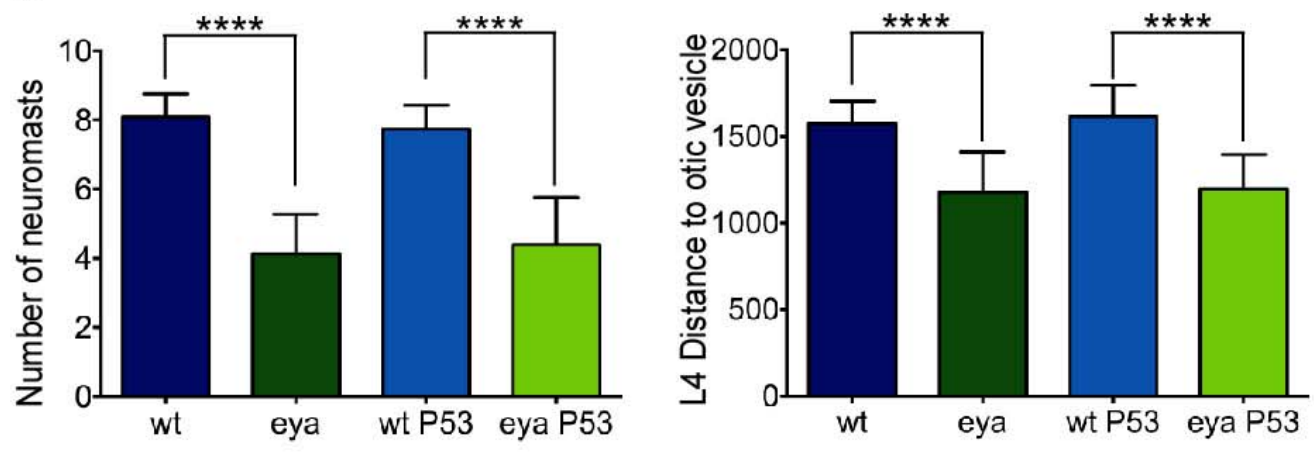

Figure 2 
bioRxiv preprint doi: https://doi.org/10.1101/2021.01.27.428404 * this version posted January 27, 2021. The copyright holder for this preprint (which was not certified by peer review) is the author/funder, who has granted bioRxiv a license to display the preprint in perpetuity. It is made available under aCC-BY-NC-ND 4.0 International license.
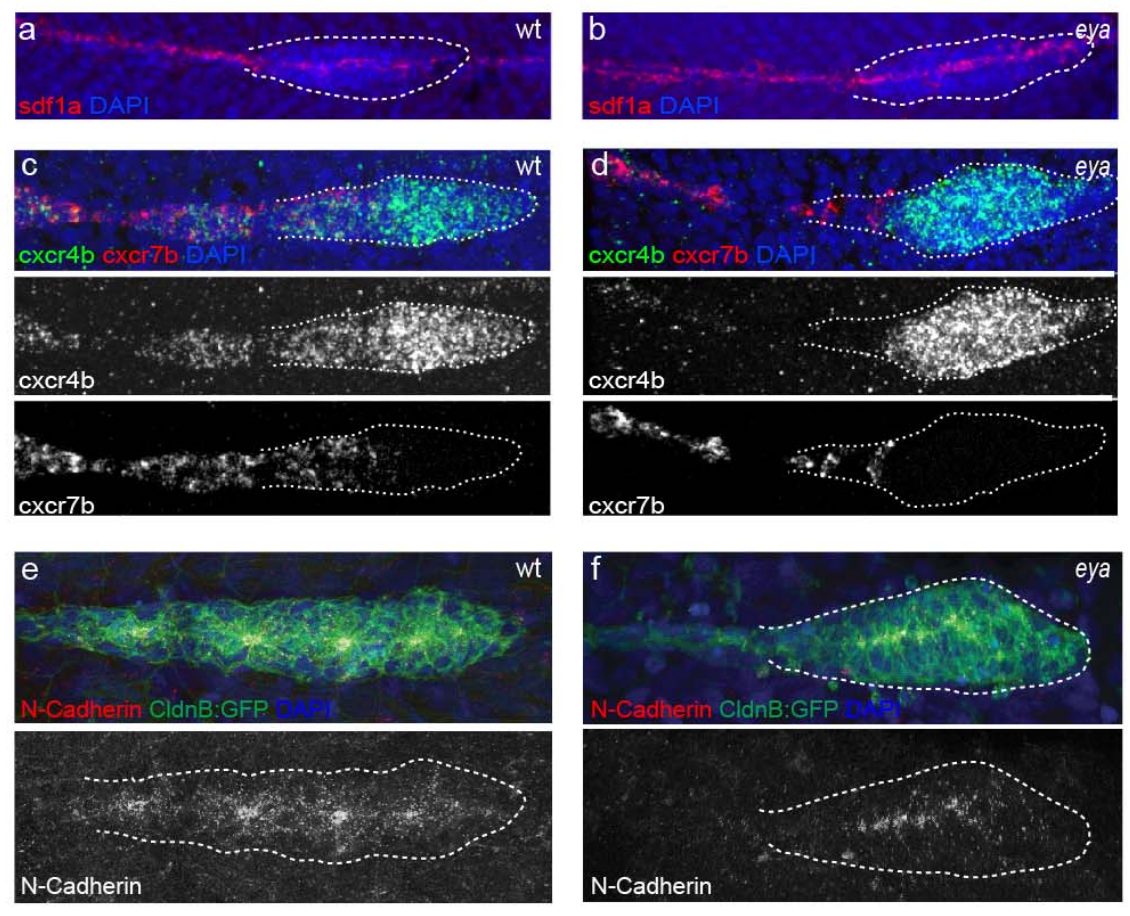

N-Cadherin

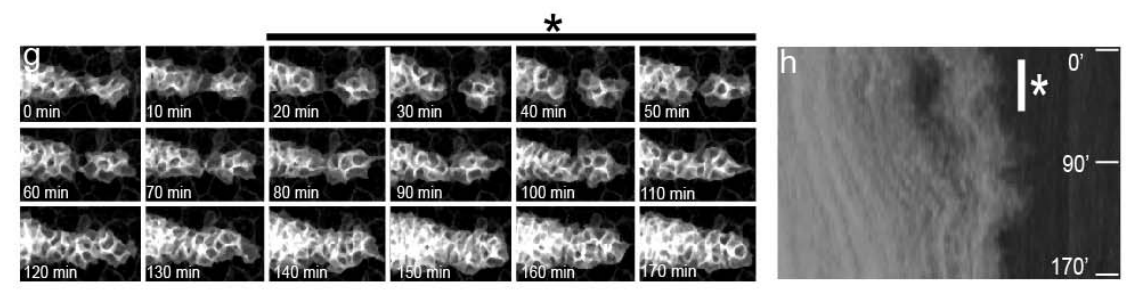

Figure 3 
bioRxiv preprint doi: https://doi.org/10.1101/2021.01.27.428404; this version posted January 27, 2021. The copyright holder for this preprint (which was not certified by peer review) is the author/funder, who has granted bioRxiv a license to display the preprint in perpetuity. It is made available under aCC-BY-NC-ND 4.0 International license.
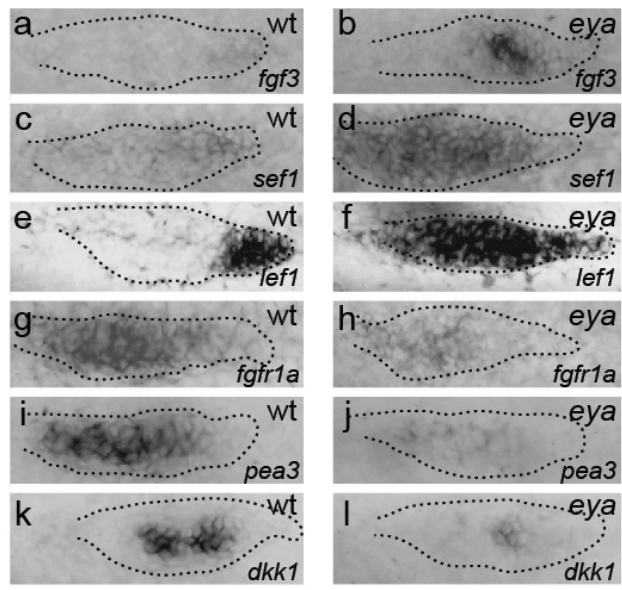

Figure 4 
bioRxiv preprint doi: https://doi.org/10.1101/2021.01.27.428404; this version posted January 27, 2021. The copyright holder for this preprint (which was not certified by peer review) is the author/funder, who has granted bioRxiv a license to display the preprint in perpetuity. It is made available under aCC-BY-NC-ND 4.0 International license.

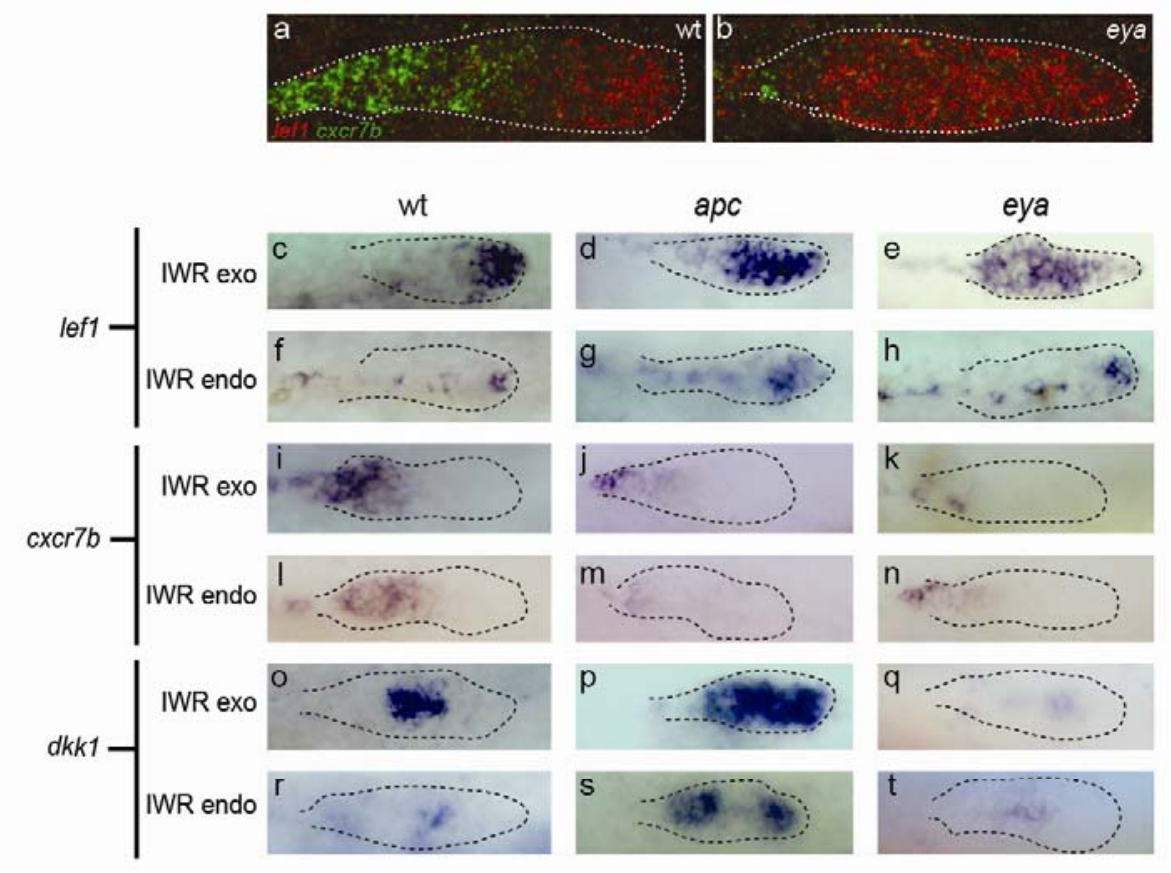

Figure 5 
bioRxiv preprint doi: https://doi.org/10.1101/2021.01.27.428404; this version posted January 27, 2021. The copyright holder for this preprint (which was not certified by peer review) is the author/funder, who has granted bioRxiv a license to display the preprint in perpetuity. It is made available under aCC-BY-NC-ND 4.0 International license.
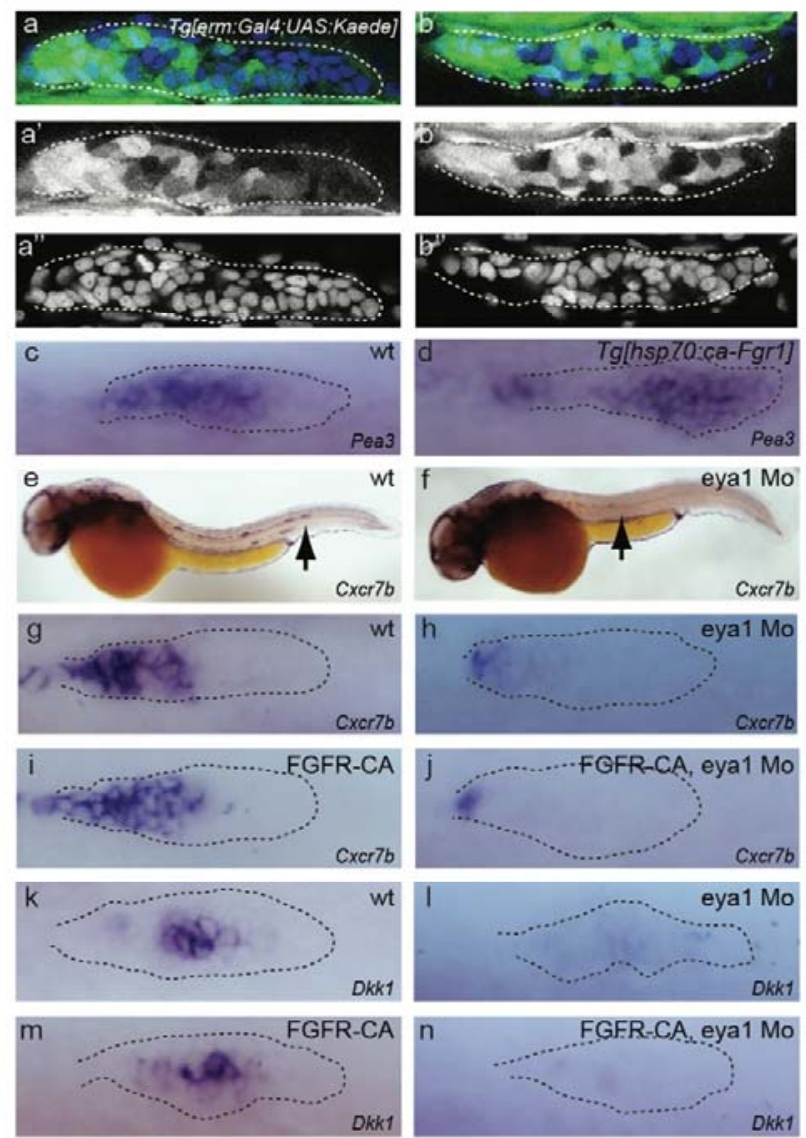

Figure 6 
bioRxiv preprint doi: https://doi.org/10.1101/2021.01.27.428404; this version posted January 27, 2021. The copyright holder for this preprint (which was not certified by peer review) is the author/funder, who has granted bioRxiv a license to display the preprint in perpetuity. It is made available under aCC-BY-NC-ND 4.0 International license.

b

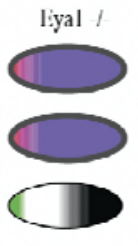

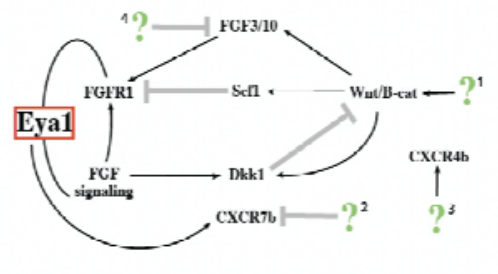

c

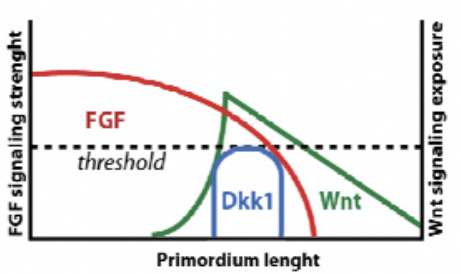

Figure 7

Primordium lenght

Figure 8 